\title{
A Case of Traumatic Bilateral Adrenal Hemorrhage Mimicking Bilateral Adrenal Adenomas
}

\author{
Min Jung Lee, Gi Ae Kim, Jung Eun Jang, Hyo In Choi, Seo Hyun Lee, \\ Gwang Beom Koh, Ga Hee Kim, Min-Seon Kim \\ Department of Internal Medicine, Asan Medical Center, University of Ulsan College of Medicine, Seoul, Korea
}

\begin{abstract}
Adrenal hemorrhages caused by blunt abdominal trauma have been frequently reported, and most of the lesions are unilateral. In contrast, bilateral hemorrhage of the adrenal glands after trauma rarely occurs in subjects with predisposing conditions such as coagulopathy, thromboembolism, and sepsis. Furthermore, bilateral hemorrhage of the adrenal glands is potentially fatal by inducing acute adrenal insufficiency. Here,a case of a 40-year-old man who developed traumatic bilateral adrenal hemorrhage after a car accident, without any predisposing condition, is reported. The spontaneous shrinkage of the bilateral lesions revealed in the follow-up abdominal computed tomography (CT) scansupported the aforementioned diagnosis. Fortunately, the patient had no clinical or biochemical evidence suggesting acute adrenal insufficiency. To these authors' knowledge, this is the first South Korean report of traumatic bilateral adrenal hemorrhage in a subject with no predisposing factors.
\end{abstract}

Key Words: Adrenal glands, Hemorrhage, Traumatic, Bilateral

\section{INTRODUCTION}

Adrenal hemorrhage caused by blunt abdominal trauma is not unusual and is mostly unilateral and right-sided. ${ }^{1,2}$ In the autopsy series, it occurred about 7-26\% after blunt abdominal trauma. ${ }^{1,3}$ However, bilateral involvement of traumatic adrenal hemorrhage has been known to be rare and usually associated with predisposing conditions such as anticoagulation therapy, sepsis, and thromboembolism. ${ }^{4}$ Bilateral adrenal hemorrhage caused by blunt abdominal trauma is clinically important as it can cause acute adrenal insufficiency leading to death, if it is unsuspected and untreated immediately. ${ }^{5}$

In a literature search, there was no case report on the traumatic bilateral adrenal hemorrhage in Korea. Therefore, we report a case of bilateral traumatic adrenal hemorrhage that developed in a man without any predisposing conditions.

Corresponding Author: Min Seon Kim, 88, Olympic-ro 43-gil, Songpa-gu, Department of Internal Medicine, Asan Medical Center, University of Ulsan College of Medicine, Seoul 138-736, Korea

Tel: (02) 3010-3245, Fax: (02) 3010-6962

E-mail: mskim@amc.seoul.kr

\section{CASE}

A 40-year-old man was admitted to our department for the evaluation of bilateral adrenal masses. He had a car accident and admitted to a local clinic three months ago. Abdominal CT taken directly after the car accident revealed splenic rupture, liver laceration, multiple rib fractures, hemothorax, and bilateral adrenal masses (Fig. 1). Further diagnostic work-up on the bilateral adrenal masses did not undergo as CT scan findings suggested bilateral adrenal masses to be post-trauma-
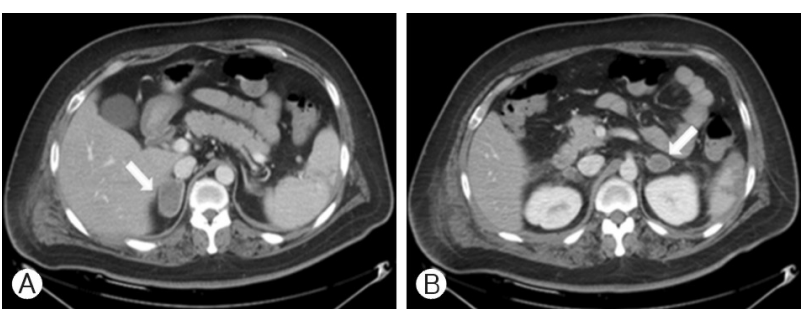

Fig. 1. Initial abdominal CT scan performed directly after blunt abdominal trauma. (A) $4 \mathrm{~cm}$ and (B) $2.3 \mathrm{~cm}$ adrenal masses without contrast enhancement are seen. 
tic adrenal hemorrhage. However, follow-up CT scan taken two weeks later showed no significant interval change in the bilateral adrenal masses. Moreover, on a careful physical examination, he had abdominal obesity, buffalo humps, and abdominal striae suggesting Cushing's syndrome. The hormonal evaluation for Cushing's syndrome was performed: Urine free cortisol level for 24 hours was $133 \mu \mathrm{g} / \mathrm{day}$ and serum concentrations of free cortisol and adrenocorticotropic hormone (ACTH) were $16.3 \mu \mathrm{g} / \mathrm{dL}$ and $124 \mathrm{pg} / \mathrm{mL}$, respectively. Thus the patient was referred to our hospital for the further evaluation of both adrenal masses and possible Cushing's syndrome.

The past medical history of the patient was unremarkable except type 2 diabetes mellitus and hypertension diagnosed 6 months ago. He had been obese since he was young. There was no remarkable finding in his family history. On admission, blood pressure was $127 / 86 \mathrm{mmHg}$ and pulse rate was 84 beats per minute. His height was $176.8 \mathrm{~cm}$, body weight was $109 \mathrm{~kg}$, and thus calculated body mass index was 35.13 $\mathrm{kg} / \mathrm{m}^{2}$. Physical examination revealed that he had cushingoid features such as buffalo hump, white abdominal striae, and abdominal obesity. He complained no specific symptom except pain around his ribs. A complete blood count showed hemoglobin $15.1 \mathrm{~g} / \mathrm{dL}$, hematocrit $44.0 \%$, white blood cell count $6,100 / \mathrm{mm}^{3}$ and a platelet count $181,000 / \mathrm{mm}^{3}$. The serum sodium, potassium and chloride levels were 135, 3.8 and 101 $\mathrm{mEq} / \mathrm{L}$ respectively. Outside initial contrast-enhanced abdominal CT scan displayed both adrenal masses with decreased attenuation (Fig. 1). Both ovoid masses were replacing normal adrenal structures. The longest diameter of the right and left mass was $4 \mathrm{~cm}$ and $2.3 \mathrm{~cm}$, respectively (Fig. 1). We re-performed hormonal evaluation for Cushing's syndrome because of a rare incidence of bilateral involvement of traumatic adrenal hemorrhage and cushingoid features. Early morning (8 A.M) blood cortisol and ACTH was $8.6 \mu \mathrm{g} / \mathrm{dL}$ and $46.2 \mathrm{pg} / \mathrm{mL}$, both of which were within normal range. Urine free cortisol level for 24 hours was $50.4 \mu \mathrm{g} / \mathrm{day}$. In the overnight dexamethasone suppression test, morning cortisol level was suppressed to be $1 \mu \mathrm{g} / \mathrm{dL}$. These hormonal data did not satisfy the criteria for Cushing syndrome. Therefore, we decided to repeat abdominal CT scan to differentiate adrenal adenomas from hematomas. On the follow-up abdominal CT scan which was done about 2 months after the initial work-up, the diameter of right adrenal mass decreased to $2 \mathrm{~cm}$ whereas that of left one was reduced to $1 \mathrm{~cm}$ (Fig. 2). A significant reduction in the size of both adrenal mass led us to diagnose bilateral adrenal masses as adrenal hematomas caused by blunt abdominal trauma. Elevated urinary cortisol excretion in initial work-up was thought to be due to post-trauma stress. Fortunately, he

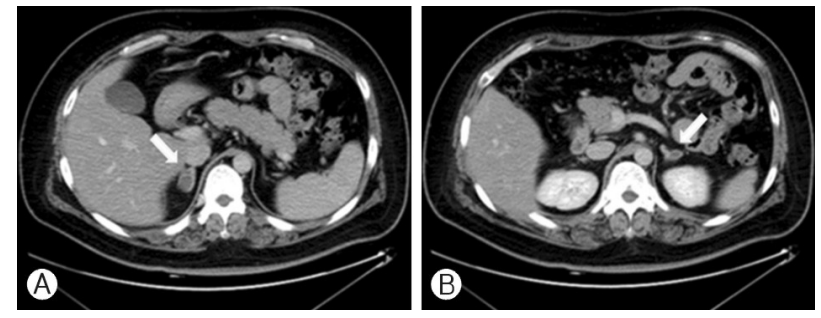

Fig. 2. Follow-up abdominal CT scan obtained 2 months later. Significant reduction of the bilateral adrenal masses is noted.

had no clinical or biochemical evidence of acute adrenal insufficiency during post-traumatic periods.

\section{DISCUSSION}

The frequency of post traumatic adrenal hemorrhage reported to be between 7 and $26 \%$ in the autopsy series and approximately $2 \%$ in CT scan. ${ }^{1,3,6}$ Post traumatic adrenal injuries occur usually unilateral. Sevitt ${ }^{3}$ firstly reported in 1955 that 14 had an adrenal hemorrhage among 50 autopsies after severe torso injuries. Among them, bilateral involvement was observed only in 3 subjects while unilateral and right adrenal gland involvement was common. In 1991, Burk ${ }^{6}$ et al. reported that 20 patients with adrenal hemorrhage were detected among the 1,120 subjects with blunt abdominal trauma using abdominal CT scans. Alike the study by Sevitt, ${ }^{3}$ injuries to adrenal gland were mostly unilateral $(17 / 20,85 \%)$ and right sided $(12 / 17,71 \%)$. Bilateral injuries were observed only in 3 patients $(3 / 20,15 \%){ }^{6}$

Several mechanisms for post-traumatic adrenal hemorrhage have been suggested although the exact mechanism is largely unknown. Sevitt ${ }^{3}$ reported that adrenal hemorrhage usually occurred in the adrenal medulla and loosely textured juxtamedullary cortex because these regions were vulnerable to damage. Therefore, after sudden compression and decompression, the vessels in those regions may be prone to rupture and thereby lead to hemorrhage. ${ }^{3}$ Regarding the mechanism for right-side preference, right adrenal gland appears to be more easily damaged because of its close opposition to the liver than the left one. ${ }^{3}$ Another explanation may come from the difference in drainage course of the adrenal veins between right and left adrenal gland. Severe compression of inferior vena cava may cause acute elevation of intra-adrenal venous pressure, leading to the adrenal hemorrhage. Since the right adrenal vein drains directly into the inferior vena cava, right adrenal gland is more susceptible to damage. ${ }^{3}$

Although traumatic adrenal injuries are usually unilateral, bila- 
teral adrenal hemorrhages have been also reported. However, most of the cases were associated with underlying predisposing conditions such as thromboembolic disease, coagulopathy, postoperative state, and sepsis rather than trauma itself. These conditions were associated with increase adrenal blood flow, resulting in adrenal hemorrhage. ${ }^{4}$ In the Korean literature, there was a case report on bilateral adrenal hemorrhagic infarction combined adrenal insufficiency due to Ebstein-Barr virus infection ${ }^{7}$ and another case report of post-traumatic unilateral adrenal hemorrhage. ${ }^{8}$ However, there has been no report on bilateral adrenal hemorrhage after blunt abdominal trauma.

Unilateral traumatic adrenal hemorrhage is usually asymptomatic and the symptoms are non-specific even if they exist. In contrast, bilateral adrenal hemorrhage can be symptomatic by causing adrenal insufficiency although it does not always happen. ${ }^{2,4,5,9,10}$ In the case series reported by Burks ${ }^{6}$ et al., one of the three patients with bilateral adrenal hemorrhage had the evidence of acute adrenal insufficiency.

Adrenal hemorrhage is usually diagnosed by imaging workup. In the CT scan, usually round or oval hematoma occupies the adrenal area. Sometimes, CT scan may show the strandlike hemorrhage infiltrating the adjacent fat or thickened diaphragmatic crues, which particularly indicate adrenal hemorrhage. ${ }^{5,69,10}$ However, it is sometimes difficult to differentiate primary adrenal hemorrhage and bleeding into a pre-existing adrenal tumor. For this reason, follow-up imaging showing a resolution of the adrenal mass in can be helpful for a diagnosis of primary hemorrhage. ${ }^{6}$ Indeed, our case showed spontaneous decrease in the sizes of both masses in the follow up abdominal CT scan. Adrenal hemorrhage usually requires no specific treatment unless it causes adrenal insufficiency. However, if there is clinical symptom suggesting adrenal insufficiency, a prompt replacement of glucocorticoid is mandatory. Delayed replacement or misdiagnosis could be lethal. ${ }^{5,10}$
We report a case of post-traumatic bilateral adrenal hemorrhage that occurred without predisposing conditions.

\section{REFERENCES}

1. Porter JM, Muscato K, Patrick JR. Adrenal hemorrhage: a comparison of traumatic and nontraumatic deaths. J Natl Med Assoc 1995;87:569-71.

2. Rammelt S, Mucha D, Amlang M, Zwipp H. Bilateral adrenal hemorrhage in blunt abdominal trauma. J Trauma 2000; 48:332-5.

3. Sevitt S. Post-traumatic adrenal apoplexy. J Clin Pathol 1955; 8:185-94.

4. Rao RH, Vagnucci AH, Amico JA. Bilateral massive adrenal hemorrhage: early recognition and treatment. Ann Intern Med 1989;110:227-35.

5. Udobi KF, Childs EW. Adrenal crisis after traumatic bilateral adrenal hemorrhage. J Trauma 2001;51:597-600.

6. Burks DW, Mirvis SE, Shanmuganathan K. Acute adrenal injury after blunt abdominal trauma: CT findings. Am J Roentgenol 1992;158:503-7.

7. Chung SC, Park SH, Lee JY, Lee SW, Jeong GH, Kim SZ, et al. A case of acute adrenal insufficiency with bilateral adrenal hemorrhagic infarction due to Ebstein-Barr virus infection in a patient with asymptomatic chronic adrenal insufficiency. J Korean Soc Endocrinol 2004;19:217-22. Korean.

8. You JS, Chung SP, Park YS, Chung HS, Lee HS, Yu JS. Isolated adrenal hemorrhage after minor blunt trauma. Am J Emerg Med 2007;25:984.e5-6.

9. Sivit CJ, Ingram JD, Taylor GA, Bulas DI, Kushner DC, Eichelberger MR. Posttraumatic adrenal hemorrhage in children: CT findings in 34 patients. Am J Roentgenol 1992;158:1299302.

10. Francque SM, Schwagten VM, Ysebaert DK, Van Marck EA, Beaucourt LA. Bilateral adrenal haemorrhage and acute adrenal insufficiency in a blunt abdominal trauma: a case-report and literature review. Eur J Emerg Med 2004;11:164-7. 